\title{
Digital Publishing, Academic Presses and the Nexus Network Journal
}

On 22 March 2001 I attended a conference organized by the Firenze University Press, the digital publishing arm of the Università di Firenze (Florence, Italy). The conference was entitled "Scholarly Communication and Academic Presses" and specifically concerned the new medium of digital publishing. My interest in participating in this conference was obviously connected to my role as editor and publisher of the Nexus Network Journal. I learned some very interesting things about online publishing that have a more or less direct effect certainly upon the authors who choose to publish their articles in the $N N J$, but also upon the $N N J$ readership.

\section{References}

First of all, as was pointed out by Anthony Watkinson, publishing consultant and visiting Professor in Information Science at City University, London, e-journals are a very new medium. He claims to have been the first to establish an e-journal in 1993, a mere eight years ago. This means that many of the issues that arise because of the newness of the medium have yet to be definitively resolved. This issues include knotty, everyday problems such as the proper form of citation of a paper published on the Internet. I have attempted to solve this problem by inserting a "Correct Citation" note at the end of each paper published in the NNJ, giving, in addition to the correct information for citing the article (author's name, title, volume and issue number, URL), a more or less accepted formal standard for the citation, so that those who want to cite an article published in the $N N J$ need only cut and paste the citation from the $N N J$ page to their own document. This is intended not only to facilitate references to articles published in the NNJ, but to encourage them.

Other issues involving the new medium are less easily resolved. One involves page content on the Internet. Digital publications are not only a translation of a traditional text into a new medium, but contain content that was not possible with print publications. Image content is one aspect of this. Animated .gif images, for instance, cannot be reproduced in print, but form a normal part of digital publications. Hyperlinks represent another normal situation. Interactive programs such as Modular Games by Slavik Jablan (produced as a CD supplement for volume 2 of the print version of the NNJ) are yet another aspect of the possibilities inherent in digital publishing. Because it is possible for the reader/user of an electronic journal to determine the content of a given page through a few clicks of the mouse, the question arises of just what page or URL he is going to cite. Virtual reality presents a further challenge. As Donald Sanders, president of Learning Sites, Inc wrote to me, his site features “...complete navigable 3D virtual spaces and in each space the user can call up linked datasets which are created to suit that particular search - different for each visitor, there is no URL for the retrieved information, nor any way (today) to bookmark the viewer's location in the world." It is clear that this takes referencing into a new realm.

Referencing is important because the number of references to articles published in a given journal determines that journal's impact rating', that is, how important the journal is to its field. Impact rating can be determined by following the number of references, a task undertaken for some journals by the Institute for Scientific Information. 


\section{Open access}

Distribution is another aspect of digital publishing that directly affects both authors and readers. Steven Harnad, Professor of Cognitive Science in the Department of Electronic and Computer Science at the University of Southampton, presented an interesting scenario in this regard. $\mathrm{He}$ pointed out that scholars do not expect to receive a royalty for publishing research work, so they give their work freely to the academic publisher. The publisher, however, interesting in making a profit, copyrights the material and publishes it in book form. Often the scholar is told by his own institution that their library has no funds to buy the book, ironically meaning that he is unable to put the book on the reserve shelf for his own students. Copyright restrictions, designed to protect the author from plagiarism, can also prevent him from photocopying and distributing his own paper in his own classes. The end result is that the author's work is held hostage" by the conventions of traditional publishing. Digital publishing is not always a better solution. Many electronic journals put only an abstract of a paper on the Internet and charge either a fee for downloading the complete article, or charge a subscription fee, giving the paid subscriber a password for access to full-text journals. Libraries are charged a licensing fee so that students and teachers can access the journal from the library terminals. Again, libraries often don 't have the funds to pay the licensing fees and students and teachers always have limited resources, so the papers published in this way are "held hostage". Prof. Harnad believes that since scholars are not earning royalties on their publications, then all scholarly works should be on everyone's desktop "for free, twenty-four hours a day, seven days a week, forever". Such a scenario is possible, if scholars routinely publish their works on the Web as part of the Open Archive Initiative (OAI). The OAI is an experimental program to provide a searchable database for the retrieval of voluntarily deposited material. Retrieval depends on the insertion of metatags that conform to certain standards (metatags are html codes that permit classification of files by information content such as author name, paper title, creation date, keywords or phrases, etc.). In effect, what is being created is a special kind of archive with its own specific search engine, to create an open resource for the scholarly community. I am now learning more about the OAI so that the NNJ can become OAI-compliant and will let you know more in the future.

\section{Long-term preservation}

I think it is important to point out that the $N N J$ is currently full-text, open access, 24 hours a day, 7 days a week. It is not, however, forever. That is, as most of our readers know, once a paper has been on the $N N J$ site for at least a year and has been published in print, so that it is part of our permanent $N N J$ archive, it runs the risk of being taken offline to make room for new material. This has caused an outcry from many readers and authors, as well as the webmasters of other sites who link to the NNJ. Naturally, the links are what the Internet is all about, and removing a paper causes a myriad of broken links. We are trying to find a solution to this problem.

The problem of a permanent archive for the $N N J$ has been discussed since its earliest days. We considered the possibility of producing a compact disc for each yearly volume. But considering how fast technology changes, there is no guarantee that a $C D$ produced today will be readable by computers ten years hence. Paper is still considered, for all its defects, the only "permanent" medium. So the $N N J$ continues to appear in print. 


\section{The limitations of digital publishing}

Although the introduction of new possibilities for content is an exciting development for publishers, the present language of digital publishing, hypertext markup language (html), presents some serious limitations. This is particularly true in mathematics, where even the latest versions of some widely-used browsers are unable to correctly read mathematical equations, rendering the paper thus published unusable. This kind of limitation means that e-journals will flourish in some fields more than others.

One way to handle the problem of mathematical equations is to convert a document in Word that includes formulas into html code, during which process the formulas will be automatically converted into .gif image files. One drawback of the importation of mathematical symbols and expression as graphics is that the .gif files can lead to a very large document, requiring long loading times. Under development is MathML, a markup language for mathematics, but it is not yet widely used or available.

A workable alternative to html is the Adobe Acrobat program that produces papers in Portable Document Format (.pdf), permitting both internal and external links (coordinated through a browser). The drawback of .pdf files is that the reader must download the file before viewing it, and use a second program (a browser) to follow external links. Thus this alternative results in a loss of much of the immediacy that characterizes the Internet.

\section{The NNJ and its community}

Another point that was brought up at the digital publishing conference was that the advent of a new medium had given rise to a plethora of new journals. Many of these, however, failed to take into account whether the particular field could support another journal, and many journal failures were blamed on a failure of the new digital medium rather than the failure of the journal itself. The question of status was brought up: are electronic journals as well-regarded as traditional print journals? Conference participants seemed to feel that there was no stigma placed on electronic journals as such, but rather that the acceptance or lack of acceptance of e-journals could be attributed to the fact that most e-journals are new journals and it takes time for any new journal to become accepted. One participant raised the question of why an author would want to submit a paper for publication to a new, unestablished journal, electronic or not, when he could submit it for publication to an already established journal of proven reputation. I was the first to answer that question, because the $N N J$ provides the perfect answer. It lies in the author finding, not the largest readership, but rather the right readership. Being an interdisciplinary journal, our readers belong to fields of mathematics and architecture. If the paper deals with, for example, the geometric analysis of an architectural monument, if it is published in either an architecture journal or a mathematics journal, regardless of how established or prestigious, the paper is only going to reach half its intended readership. This was the case with the first papers detailing fractals, which were published in a journal for meteorologists and so were unknown to mathematicians for some time, a story that has always stuck in my mind.

But what determines whether a new journal becomes an established journal or a failure? This is determined by how many papers are submitted for publication. If enough material arrives, then with time the journal will become established. If not enough papers are submitted for publication, then the journal will have to close its doors. In effect, as Steven Harnad expressed it, "authors vote with their papers". I am very happy to say that authors are voting for the Nexus Network Journal, because material continues to arrive. 
I think what is so exciting about the Nexus Network Journal is that we are not only establishing a new journal, exploiting the possibilities of a new medium and diffusing scholarly material of high quality, but we are defining a new discipline in the process. Before the beginning of the Nexus conferences in 1996 (organization actually began in 1995), there was no field of architecture and mathematics as such, whereas now, only 6 short years later, we not only have a field, we have a community as well. Of course for now the $N N J$ is the only journal in its field. How would I feel if another, competing journal were established? I would feel GREAT because that would really mean that the field of architecture and mathematics had come of age.

The NNJ authors and readers are partners in pioneering the new medium of digital publishing, as well as in the establishment of a research community and a new interdisciplinary field of research. I 11 be looking forward to telling you about our process of growth in future issues.

Kim Williams, Editor-in-Chief

8 NEXUS NETWORK JOURNAL - VOL. 3, NO.1, 2001 